\title{
Agent-based and multi-agent simulations: coming of age or in search of an identity?
}

\author{
Dwight Read
}

Published online: 30 July 2010

(C) The Author(s) 2010. This article is published with open access at Springerlink.com

\begin{abstract}
The promise of agent-based for explicating properties of social systems has not yet been fully realized. Agent models sometimes provide only a veneer of, rather than substantive engagement with, social behavior. The problem will be illustrated with Axelrod's model for evolution of ethnocentrism (a biological model) versus Schelling's model for spatial segregation based on preferences (a cultural model). The examples show the need to incorporate both the biological and cultural basis for behavior through a schema that includes behavior based on cultural/cognitive processing of information and behavior based on biological/cognitive processing of information. An example of an agent-based model that implements decision making in this manner is discussed. The model accounts for heterogeneity in behavior outcomes and leads to two main predictions: (1) small scale, hunter-gatherer societies in resource scarce environments will have stable adaptations less affected by variation in resource abundance in comparison to groups in resource rich regions where inter-group conflict is more likely and (2) the relationship between community size, population size and administrative complexity will have two distinct patterns, one for patrilineally organized societies and the other for matrilineally organized societies. Both predictions have been verified empirically.
\end{abstract}

Keywords Agent-based modeling - Social simulation · Culturally based decision making $\cdot$ Birth spacing model $\cdot$ Paramount states $\cdot$ Administrative hierarchy

Social Simulation Workshop, International joint Conference on Artificial Intelligence, Pasadena, CA, July 11, 2009.

D. Read (凶)

Department of Anthropology and Department of Statistics, UCLA, Los Angeles, CA 90095, USA

e-mail: dread@anthro.ucla.edu 


\section{Introduction}

The utility of agent-based modeling for the social sciences seems almost self-evident. Social systems, by their very definition, involve interacting agents with a multitude of interests, goals, and intentionalities. As participants in a social system we do not see ourselves and those with whom we interact as acting in easily predicted manners and we depend upon a variety of social institutions and role-systems to make the complexity of social interaction more manageable. In comparison to the agents in most other mammalian social systems, we are highly individualistic in the sense we are capable of a wide range of behaviors according to the context in which we interact. For analytical purposes, we divide this range of behaviors into categories such as religious, economic, political, and kinship behavior and academically we specialize in the analysis of, and develop theories for, particular kinds of behavior. As anthropologists are quick to point out, these divisions may be analytically useful but sometimes imply a separation in kinds of behaviors that do not reflect the reality on the ground. Because agent-based modeling lends itself to a more holistic approach to simulation of social systems without imposing boundaries, many have noted the integrative aspect of agent-based modeling as one of its strengths: "By focusing attention on the actors in social and environmental settings, agent-based models may have an important role to play in integrating theories from psychology, economics, sociology, geography and elsewhere.... provoking theory development and also enabling testing of theories." (O'Sullivan 2008, p. 546).

Beyond an integrative aspect, agent-based modeling makes it possible to engage in experimental methods that are the hallmark of the physical sciences. We are "provided for the first time the possibility of using experimental methods with social phenomena" (Gilbert 2007, p. 115), a theme taken up by several of the papers in the workshop: "multi-agent simulation offers an attractive alternative to field or laboratorybased studies of group decision making mechanisms" (Zappala and Logan 2009, p. 2) and "AmISim [Ambient Intelligence Simulation] provides a simulation framework which can test scenarios that would be impossible in real environments." (GarciaValverde et al. 2009, p. 11).

Despite the very real advantages offered by social simulations, the social sciences have been slow to adopt multi-agent modeling as part of the methods of the social sciences. As one commentator has noted recently,

"Complex adaptive systems models have the potential to transform social science. With them scholars can construct and analyze models that include real world features such as networks, adaptation, heterogeneity, and interactions. ...The notion that social simulation models can improve social science should be non controversial, thus, my puzzlement over the current state of affairs. While social simulation has made inroads ... it has yet to become a core methodology of social science" (Page 2008, emphasis added).

This theme was taken up by several of the workshop papers. One paper suggested that there has been an overemphasis on agent-based modeling of the supposed rationality of humans and not on humans per se: "much of the potential [of agent-based modeling] is underdeveloped because agent based approaches historically have been 
centered on rationality but not humans." (Zhang and Leezer 2009, p. 1). The distinction was made very graphically in another paper through noting that humans resort to "bullshit" and not rationality as a way to manipulate social interactions: "different situations. . . in human society, provide strong individual incentives for bullshit. Overall, our analysis is meant to identify some particularly troublesome issues regarding reasoning in social context." (Caminada 2009, p. 1).

These comments indicate that despite the optimism for social simulations, the human side of what is involved in social interactions is underdeveloped. Even for areas as well studied as the concept of norms in human behavior, attempts to simulate normative behavior are still in their early stages:

"This paper investigates to what extent normative agent-based models are able to capture the role theoretic concept of norms. Three methodological core problems are identified: the question of norm transmission, normative transformation of agents and what kind of analysis the models contribute. ... However, the degree of resolution of intra agent processes remains too low for a comprehensive understanding of normative behaviour regulation... [and] an investigation of the recursive impact of inter- and intra-agent processes is still in its fledgling stages." (Neumann 2008, emphasis added).

New approaches may resolve some of these issues. One of the workshop papers addressed ways to model changes in norms in a linguistic context: "We have presented an approach to study consensus [norms] under constraints, and have shown that we can model phenomena of sound change such as vowel shifts. However, the same approach can, in principle, also be applied to any situation where we have a system of constrained variables over which consensus must be achieved." (Lakkaraju et al. 2009, p. 4). But more than just new simulation approaches are needed. We need to examine the models we use when simulating human behavior and to see if they are adequate, especially with regard to the cultural side of human behavior.

\section{Biological versus cultural modeling of social behavior}

\subsection{Biological model for the evolution of ethnocentrism}

We can illustrate the question being raised here with a model developed by Robert Axelrod and Ross Hammond (Axelrod and Hammond 2003) for the evolution of ethnocentrism. The model is very simple, yet appears to have far-reaching implications about the evolution of ethnocentric behavior. At first glance, it appears to address the human side of social behavior by using terms such as ethnocentrism.

In the model, agents have a trait value for group membership (one of four colors) and a trait value (cooperate or defect) for each of two interaction strategies: (1) action taken with same-group members and (2) action taken with other-group members. All three trait values are inherited by an offspring. Agents with an ethnocentric interaction strategy are defined as those who cooperate with same-group and defect with other-group agents. The simulation begins with a cohort of agents, with a uniform distribution of trait values, migrating randomly to a region. Agents reproduce 
according to initial fitness values modified in accordance with the outcome of Prisoner's Dilemma games played between agents and spatially adjacent agents. Agents die according to a fixed mortality rate. ${ }^{1}$

The goal of the simulation is to explore conditions leading to evolution of the ethnocentric interaction strategy. They find that "the simulation results show that the ethnocentric strategy becomes common even though there is no explicit bias for it in the model ...76\% of the agents have the ethnocentric strategy" (Axelrod and Hammond 2003 , p. 11), which leads them to conclude "that in-group favoritism can overcome egoism and dominate a population even in the absence of reciprocity and reputation, and even when 'cheats' (mimics) need to be suppressed" (Axelrod and Hammond 2003 , p. 12). On the face of it, the simulation appears to show that a simple model accounts for the evolution of ethnocentrism, a very human behavior.

\subsubsection{Conceptual problems with the simulation}

The results are less impressive, though, when we realize that the Prisoner's Dilemma game is actually implemented as two independent games (with the same payoffs) for each of the two strategies (see source code [Wilensky 2006] for the simulation). Game 1 is played when interacting with a same color agent and Game 2 is played when interacting with a different color agent. Since these are independent events, Prob(ethnocentric strategy) $=$ Prob(cooperate with same color agent) $\times$ Prob(defect with other color agent). For example, using the spatial condition that an offspring resides adjacent to a parent, Prob(cooperate with same color agent $)=0.9$, Prob(defect with other color agent $)=0.84$ and Prob(ethnocentric behavior $)=0.9 \times 0.84=0.76$ (all three probabilities from Axelrod and Hammond 2003, p. 11). In other words, it is not the frequency of the ethnocentric strategy that is evolving but the frequencies for each of the two strategies, "action taken with same-group agents" and "action taken with other-group agents."

The evolution of these two strategies is driven by the spatial structure induced under the assumption that an offspring is adjacent to its parent. This assumption leads to agents of the same color being distributed in spatial clusters and all agents in a cluster having the same behavior due to trait inheritance. When an agent interacts with an adjacent agent of the same color, both are from the same cluster and so both have the same behavior when playing the Prisoner's Dilemma game: either both cooperate or both defect. Under these conditions, the game payoffs favor the agents with the altruistic behavior, "always cooperate" when interacting with an agent from the same group: "The success of altruism is due to local reproduction and interaction where altruists are likely to meet others with the same genes" (Németh and Takács 2007).

When interacting agents have different colors they are from different clusters. The distribution of clusters by color is spatially random since the agents whose initial location under migration is random determine the clusters. Hence for this Prisoner's Dilemma game, agents are essentially interacting randomly with respect to color and

\footnotetext{
${ }^{1}$ The simulation is included in the library of social science models distributed with NetLogo (Wilensky 1999).
} 


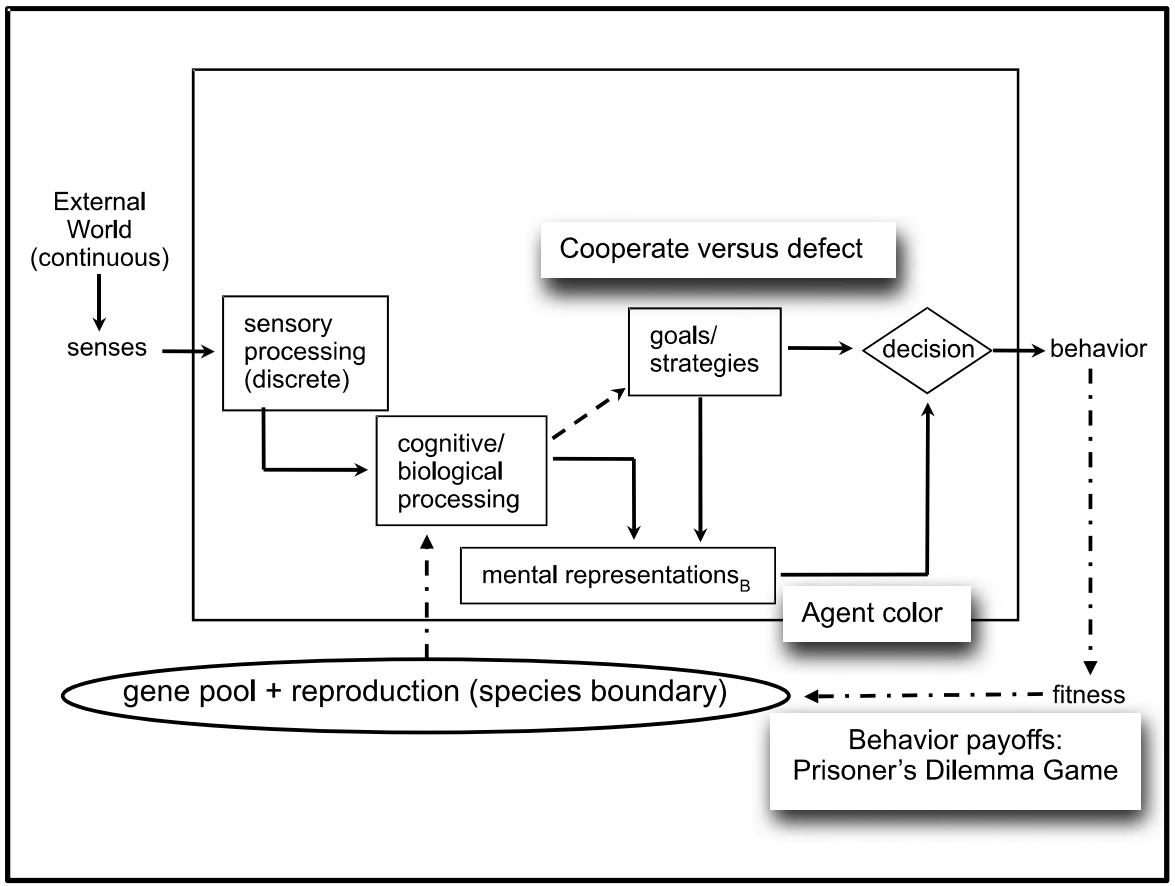

Fig. 1 Schema for behavior based on cognitive/biological processing of sensory information. Shadowed text refers to simulation properties. See text for details

behavior. As is well known, under these conditions the defect strategy will win out under Prisoner's Dilemma payoffs.

If, instead of being adjacent to parent, the offspring are located randomly with respect to parent in the simulation, what is being called ethnocentric behavior should not evolve since all agents would now be interacting randomly with respect to color and behavior. Not surprisingly, under these conditions there is "only 5\% cooperation in the populations as a whole" (Axelrod and Hammond 2003, p. 14).

Although the simulation is couched using terms such as ethnocentrism, the underlying structure of the simulation does not relate to ethnocentrism as a trait but simply as a label for the intersection of "cooperate with same color agent" and "defect with other-color agent." The frequencies for these two strategies are driven by the two independent Prisoner's Dilemma games with outcomes structured by a demographically induced spatial distribution for the agents.

\subsubsection{Simulation uses a biological schema for behavior}

Now step back from the details of the simulation and consider the implicit model for human behavior underlying the simulation. As shown in Fig. 1, it is a biological schema for behavior without a cultural component, hardly a satisfactory model for ethnocentric behavior. Beginning on the left side of Fig. 1, the schema begins with external signals processed by the sensory apparatus and then subjected to cognitive 
processing, with output a mental representation. In the simulation, an (implicit) mental representation for an agent is the color and spatial location of another agent. In the schema, goals and strategies are used to identify the relevant mental representations when formulating a behavior aimed at satisfying a goal (in the simulation, agents have a reproductive goal mediated through interaction with an adjacent agent using a Prisoner's Dilemma game and so agents pay attention to the color and spatial location of other agents). The schema shows a connection from cognitive processing to goals and strategies; goals and strategies are not fixed but may change according to sensory information received and cognitively processed by the agent. The goals and strategies and the mental representations are then evaluated and lead to a decision as to what behavior to employ (e.g., play a Prisoner's Dilemma game with an adjacent agent). Agent behaviors have consequences for fitness values (via modification of the fitness value by the payoffs from the Prisoner's Dilemma game) and the fitness values feed back in a standard way to the frequencies in the gene pool for the agents. The gene pool has a species boundary (implicit in the simulation). In sum, the simulation is essentially a standard biological model for a phenotypic trait with biologically based behaviors included as part of the phenotype. Culture plays no role in the schema and so ethnocentric behavior is assumed to be culture free, a questionable assumption.

\subsection{Cultural model for the evolution of spatial segregation}

Now compare this simulation to the now-classic Schelling simulation model for the emergence of spatial segregation due to agent decision-making based on the likelihood of moving to a new location as a function of an agent's degree of happiness with her/his current neighborhood. Happiness is based on the degree of similarity (however similarity might be conceptualized) with one's neighbors. The simulated decision-making behavior is based on assumptions about social behavior that has theoretical and empirical support, as noted by one of the workshop papers: "social comparison is a general cognitive process underlying social behavior" (Fridman et al. 2009 , p. 1). In addition, similarity is measured using information available to a person in the real world and the simulation uses a plausible decision model based on taking action when a trait value exceeds an individual-specific threshold value.

The simulation has been widely cited and used for teaching purposes due to the seeming plausibility of the assumptions of the simulation, hence the outcomes of the simulation relate to real-world phenomena. The simulation shows that segregation can be self-organizing and emergent even absent any imposed constraints on the spatial location of agents. Even with decisions that would be seen by most to be unbiased-stay in a neighborhood when even $60 \%$ of one's neighbors are unlike oneself-segregation emerges. The outcomes of the simulation challenge conventional wisdom that has attributed urban segregation to imposed restrictions:

"The implication of much commentary on urban areas is that the urban fabric would quickly become integrated if discrimination and other constraints on residential opportunities were eliminated (Zubrinsky and Bobo 1996). The preference and social distance work both in its mathematical and agent-based forms, and the survey results raise serious questions about this view (Fossett 2006). They suggest that mere tolerance and the absence of virulent housing 


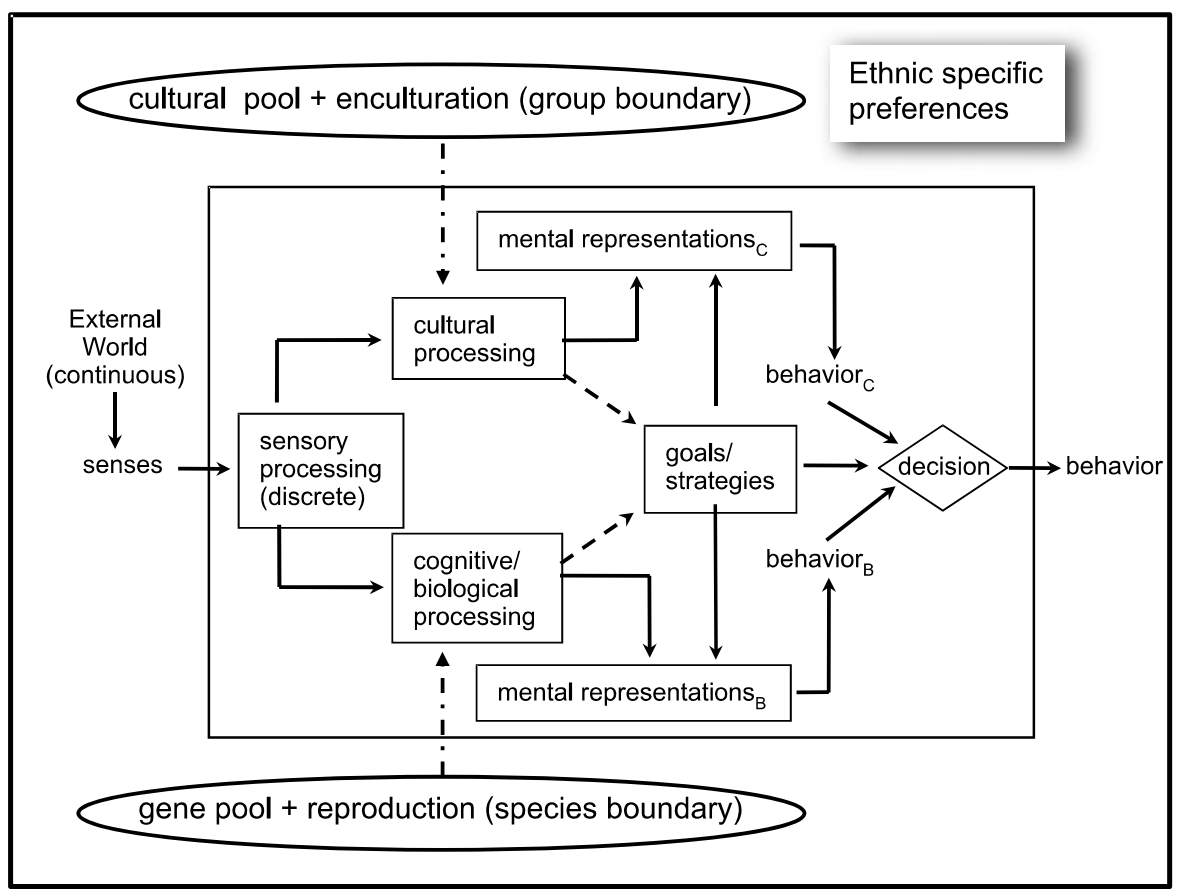

Fig. 2 Schema for behaviors based on either cognitive/biological or cultural processing of sensory information. Shadowed text refers to simulation properties. See text for details

discrimination will not produce integration under prevailing patterns of ethnic preference, at least, not in the short run." (Clark and Fossett 2008, p. 4114, emphasis added).

Contrary to the simulation by Axelrod and Hammond (2003), Clark and Fossett argue, and the Schelling simulation demonstrates, that preexisting ethnic preference is a driving force for the segregated spatial structure found in urban context and not the reverse.

\subsubsection{Cultural schema for behavior}

We can characterize the Schelling simulation with a behavior schema that includes a cultural component (see Fig. 2). In this schema, a cultural processing component, acting in parallel with the cognitive/biological processing component, assigns cultural meaning to external phenomena. By cultural meaning is meant the common meaning shared among those enculturated in the same cultural system. This contrasts with the individual meaning of external phenomena provided through cognitive/biological processing of sensory data and where shared meaning occurs only in a statistical sense.

We can illustrate the difference in the two kinds of meaning with a simple example of public spaces with lawns and walkways for pedestrians. Often lawns have a dirt path created by agents following the shortest path between two points rather 
than the walkway culturally marked as the appropriate walking route. That is, we sometimes act according to a cognitive/biological processing system biased towards following the shortest path or sometimes according to a cultural processing system biased towards following the path designated as the appropriate path even when it is longer. The biological processing system reflects pan-species evolutionary selection for visual processing systems biased towards following a straight-line path when physically feasible. When we use the walkways, we are using our cultural processing system that recognizes the walkway as having the cultural meaning "this is the proper place to walk," hence we are acting in accordance with the shared, cultural meaning of a walkway. The latter contrasts with individual calculation of the shortest path using cognitive/biological processing. Different individuals may make the same calculation due to their shared biological heritage, which leads to many individuals coincidentally following the same shortest-distance path, thereby leading to the formation of a dirt path across the lawn.

When we observe individuals arriving at a point where a choice can be made between a shortest distance path and a longer walkway, some individuals decide one way and others decide the other way. If we watch the same individual over several days, that person may take one route on some occasions and the other route on other occasions-perhaps for reasons such as "I'm late, so I'll walk across the lawn," or perhaps for no particular reason.

This simple example underscores the fact that, as culture-bearers, we have two, distinct systems for the processing of the sensory information we receive from the external world as indicated in Fig. 2. This also implies that we are part of two systems with different boundaries: a biological system with a species boundary and a cultural system with a social boundary determined by those who are enculturated into the same cultural system.

Mental representations must now be divided into biological versus cultural representations (as indicated by the subscripting in the schema). Sometimes the two may be the same, but other times they are widely divergent, as with the culture-specific concept of humanness that has no biological counterpart. These two systems can each lead to a possible behavior, hence we must also have a decision system that adjudicates between the behaviors - this can be seen in our decision on one day to walk across the lawn and on another day to follow the walkway.

\subsubsection{Schelling simulation and the cultural schema for behavior}

The Schelling simulation fits with this schema through the neighbors (external phenomena) being evaluated as similar or dissimilar, which may be based on culturally identified attributes for making such a comparison. We engage in public discussions regarding what are proper or improper attributes for making such an evaluation and what weighting should be placed on them. The Schelling model captures the current outcome of such a discussion through the parameter value for the degree of similarity agents needed for staying put. Though the decision-making involves an emotion (happiness due to similarity of neighbors) related to cognitive/biological processing, the attributes affecting the emotional state for an agent may depend on cultural criteria. The cognitive/biological processing might lead to behavior $\mathrm{B}=$ "stay put" (since this 
behavior has less cost than moving to another location and no fitness benefit accrues in the simulation from randomly moving to another location), while at the same time the cultural processing may lead to the behavior $\mathrm{C}=$ "move to new location" (since not all of one's neighbors are similar to oneself) and a decision is made between these two behaviors through an adjudication process. ${ }^{2}$ In the simulation, the adjudication is done by comparing the degree of unhappiness due to dissimilar neighbors with a threshold value: chose behavior $r_{B}$ if unhappiness $<$ threshold value, otherwise choose behavior $_{C}$.

\section{Implications and predictions of a cultural based simulation for three spatial patterns and forms of social organization}

Now we want to use the cultural schema to simulate the implications of culture-based behaviors. The context for the simulation is the !Kung san, a hunter-gatherer group that inhabits the northwestern part of Botswana in Africa. In the simulation, agents act in accordance with the culturally framed comments !Kung san women make about children and healthy families. The goal is to explore the consequences their comments for behavior relating to birth spacing and through that for emergent group properties such as a stabilized population size (Read 2003). We will then apply the model to three different spatial configurations for social units and access to resources that characterize hunter-gatherer, patrilineal and matrilineal societies, respectively. In each case, we will make non-trivial predictions from the model that are then verified by ethnographic data.

\subsection{Decision model for birth spacing}

Like other hunter-gather groups, the !Kung san are divided into social units, which we can refer to as residence groups, that are the locus for day-to-day activities. A residence group is made up of around 5-6 families, or about 25-30 persons. Spatially associated with a residence group is a region they refer to as a $n$ !ore. All members of the residence group have the right to hunt and forage for resources in the $n$ !ore and have access to the resources in the n!ore of another residence group through culturally constituted kin relations expressed through a kinship terminology. This implies that, for simulation purposes, we can consider the population as a whole without dividing it into residence groups.

For the population as a whole, we need to distinguish between absolute carrying capacity, $K$, defined as the maximum number of individuals that can be sustained keeping fixed the mode of resource procurement, and the observed carrying capacity, $K^{*}$, the equilibrium density (if any) that ensues from the group's mode of resource procurement and decisions made about birth spacing. With fixed area from which resources are obtained, we may measure $K^{*}$ and $K$ as population sizes. The goal of the simulation is to relate $K^{*}$ to behaviors derived from what woman say about having families and children.

\footnotetext{
${ }^{2}$ Under appropriate circumstances, natural selection can favor a biological behavior for staying put or moving to a new location based on the degree of similarity to adjacent conspecifics. In keeping with the goal of the Schelling model, we are assuming the behaviors are not biologically grounded.
} 
When asked, they say that "[t]hey want children, all the children they can possibly have...," which leads to decrease in birth spacing. At the same time, they want healthy families and that acts as a constraint, for "they explained that they cannot feed babies that are born too close together.... A mother had not enough milk to sustain completely two infants at the same time." This concern leads to increase in birth spacing. These two interests are mediated through the absolute time/energy constraint faced by each woman: "to carry a third child and the food she gathers would be practically impossible for those small women." The women use intensive, on demand nursing, which is also a highly effective method for birth control. They do not conceptualize nursing as a means for birth control, though, but see the duration of nursing as related to the health of a child: "they believe a child must have strong legs, and it is mother's milk that makes them strong ... a child needs milk till he is three or four years old at least" (all of the quotes are from Marshall 1976, pp. 166, 168). Their perception does not have a biological basis, hence they are justifying the practice of nursing a child for several years while leaving open when a woman will stop nursing since "have strong legs" does not relate to any specific age for nursing to stop. In brief, each woman is making "selfish" decisions based on her perception of what is best for herself and her family. There is no indication that the women are making any kind of group-oriented, altruistic decision.

From their comments, we form a simple, birth spacing decision model based on a woman's current time/energy cost for foraging and parenting, using the following two simplifying assumptions:

(1) cost of parenting $=$ (number of infants $) \times($ cost of parenting per infant $)$ and

(2) cost of foraging is proportional to the population size, where an infant is defined as the time span from birth to cessation of nursing, the age range requiring the greatest time/energy invested by a mother.

The decision rule is:

Decision Rule: Become pregnant if Total Cost $=$ Cost of Parenting + Cost of Foraging $<E_{\max }$, where $E_{\max }$ is the maximum timelenergy that can be spent on foraging + parenting.

No attempt is made to calculate realistic values as the goal of the simulation is to explore relative and not absolute effects. For fixed $K$, the simulation results can be summed up as follows (see Read 1998, 2003 for details). First, $K^{*}$ varies directly with the perceived cost of parenting. Cost of parenting beyond biological requirements typically includes a cultural component, so $K^{*}$ varies directly with change in cultural notions of what parenting involves. Second, $K^{*}$ varies inversely with maximum cost, $E_{\max }$, so to the degree $E_{\max }$ is culturally specified, $K^{*}$ is also culturally specified. Thus, the magnitude of $K-K^{*}$, the degree to which a population is shielded against stochastic variation in resource abundance, is determined by factors that have cultural specification and is not simply a consequence of the mode of resource procurement. We now apply the model to three patterns for the spatial relationships of group members and social units to resources. The three spatial patterns characterize societies with a band-level of organization (small scale hunter-gather societies), a patrilineal mode of social organization and a matrilineal mode of social organization. 


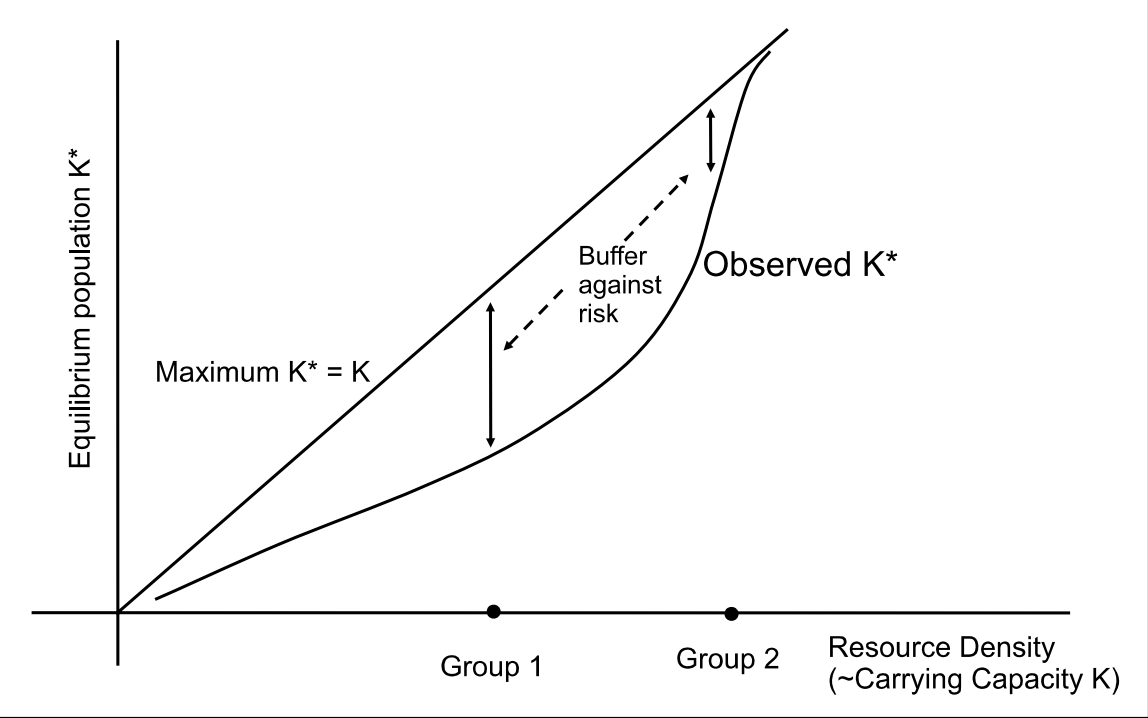

Fig. 3 Predicted equilibrium population $K^{*}$ versus absolute carrying capacity $K$. Predicted curve has a C shape

\subsection{Spatial Pattern 1: definition and predictions}

Definition The society is made up of residence units based on a small number (4-7) of families. All members of the society have access to the resources in the region occupied by the society, either directly or indirectly through kin relations. Population growth increases the total number of persons accessing the same resources.

Spatial Pattern 1 is typical of hunter-gatherer societies. We will now make predictions that relate to differences in the spatial density of food resources for huntergatherer societies in different environmental conditions. We compare two huntergatherer groups, one in a resource rich environment and the other in a resource poor environment. The cost of foraging per day in a resource poor environment increases at more than a proportional rate with the difference in resource density since foragers in resource poor environments have at least the extra cost per day of traveling throughout the increased spatial area over which they must forage. Thus $K^{*}$ will decrease more rapidly than just in proportion to the change in $K$. Ultimately, though, as resource density goes to zero, $K^{*}$ must approach $K$ since in the limit of a region with no resources, $K=K^{*}$. In regions with high resource density, reduction in fertility through increased spacing of births may be insufficient to reach an equilibrium, $K^{*}$, and instead the population size will continue to increase until it is limited by $K$; that is, by Malthusian parameters such as warfare, disease, starvation. These observations predict that a graph of $K^{*}$ against $K$ will have a $\mathrm{C}$ shaped curve in relationship to the linear change of $K$ with resource density as shown in Fig. 3. The C curve also implies that conflict will be more likely in resource-rich environments where $K^{*}$ is bounded by $K$. 


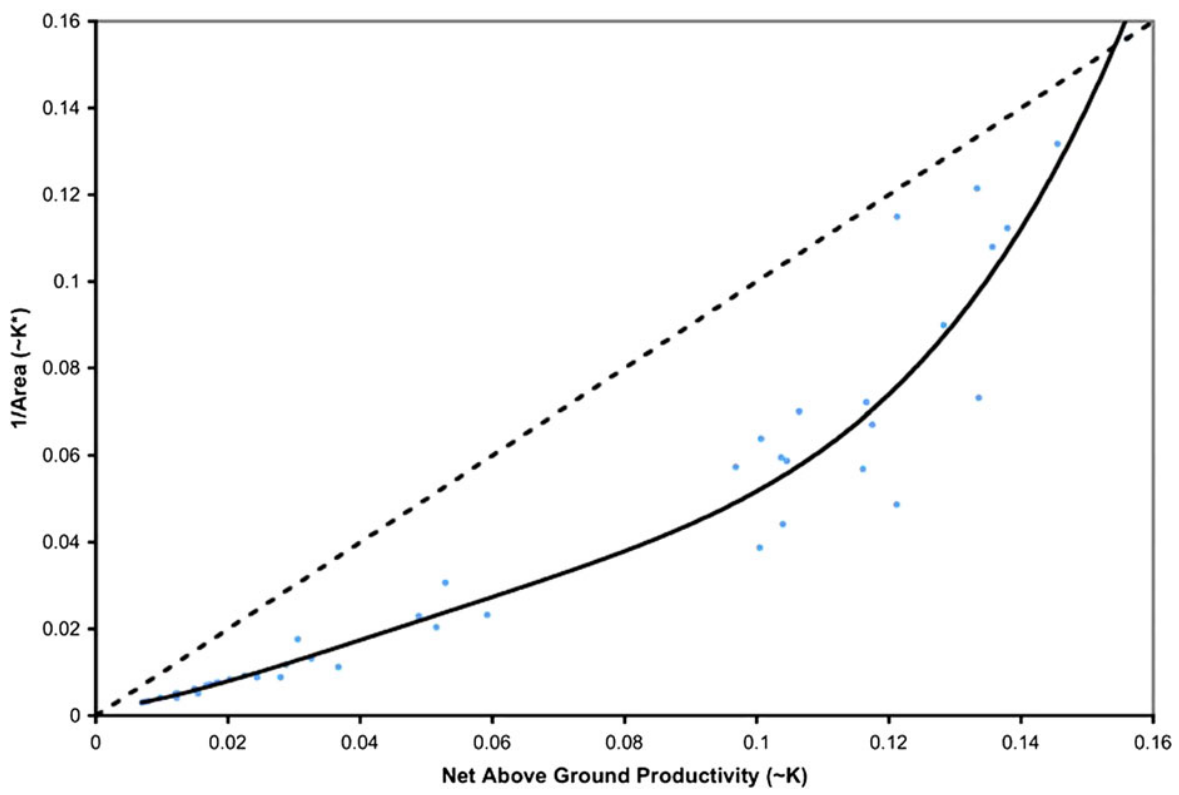

Fig. 4 Observed data for Australian hunter-gatherer groups. $K^{*}$ is proportional to 1/area, where area is the total region used by a group, since population size has a modal size among hunter-gatherer groups and so $K^{*}$ varies inversely with area. $K$ is proportional to net above ground productivity. The curve is a best fitting 2nd degree polynomial. Data are from Binford (2001)

Data on hunter-gatherer groups in Australia corroborate the predicted $\mathrm{C}$ shape (see Fig. 4). The prediction about conditions leading to conflict is also supported: "[W. Lloyd] Warner found multiple types of Murngin [an Australian hunter-gatherer group] conflict ... much of this lethal violence occurred in collective battles and raids between rival groups ... they inhabited a rich ecological environment" (Knauft 1996, p. 85, emphasis added). Conflict like this has a long history in Arnhem Land, the northern, resource rich area occupied by the Murngin (Taçon and Chippindale 1994), including correlation between increase in resource availability and conflict: “... climatic changes rendered Arnhem Land more ecologically plentiful 4,000 to 6,000 years ago... [rock] art style during this period exhibits a much greater incidence of collective armed conflict..." (Knauft 1996, p. 86).

\subsection{Spatial Pattern 2: definition and predictions}

Definition The society is made up of communities (e.g., villages) made up of a moderate number (10-15) of families. Each community is based on a single social unit that has corporate control over a resource base for the community members. Social units (hence communities) fission in response to internal conflicts due to population growth. Fissioning leads to increase in the total spatial spread for the geographic location of social units since the resource base of a social unit is not split when fissioning takes place. 
Now consider some of the implications the model for birth spacing has for societies organized in accordance with spatial pattern 2. Social unit fissioning (which limits the size of social units) in conjunction with corporate control of resources "shields" females from population growth feedback affecting the time/energy women spend on resource related tasks. This implies, according to the model, that birth spacing will not increase with population growth for the society as a whole and the net fecundity rate will always be positive. Consequently, $K$ and not $K^{*}$ limits the total population size. Fissioning leads to a proportionate increase in the total number of social units and new units require new localities for resources, but the spatial spread of social units is eventually limited by neighboring societies, hence population increase will lead to within society, inter-unit conflict over resources. Segments of the society can "hive off" as a consequence. A social unit, though, tends to be a stable, socially cohesive unit. The total number of social units that can be maintained in a single society is eventually limited by the relative autonomy of social units, lack of centralized political structure, and society-level fissioning along conflict cleavages that may arise between groups of social units. Patrilineal societies are a canonical example of a society with this spatial pattern.

We make the following predictions.

Prediction 1 Societies will not be large and will have social units with population size around 50-75 persons.

Prediction 2 Administrative organization will be limited and vertically shallow since social units do not require a hierarchy for internal cohesion of a kin-based social unit and is activated primarily at a local level for adjudicating conflicts between social units.

We will verify these predictions in conjunction with the predictions for Spatial Pattern 3.

\subsection{Spatial Pattern 3: definition and predictions}

Definition The society is made up of communities composed of several social units. A social unit may be sub-divided and located in more than one community. Social units are corporate units within the community. Social unit fissioning occurs within a community and does not lead to fissioning of communities.

As with Spatial Pattern 2, a corporate unit controls access to resources and so women are shielded from feedback from population growth. Thus birth spacing will not increase with population increase and so the society population size is limited by $K$ and not $K^{*}$. Population growth and fissioning of a social unit within a community leads to intra-community and not inter-community conflict. Community-level administrative hierarchy develops as a way to deal with both intra-community conflict among social units within a community and with relations between communities when social units are distributed in more than one community. The population size of the society increases with population growth of communities and there will 


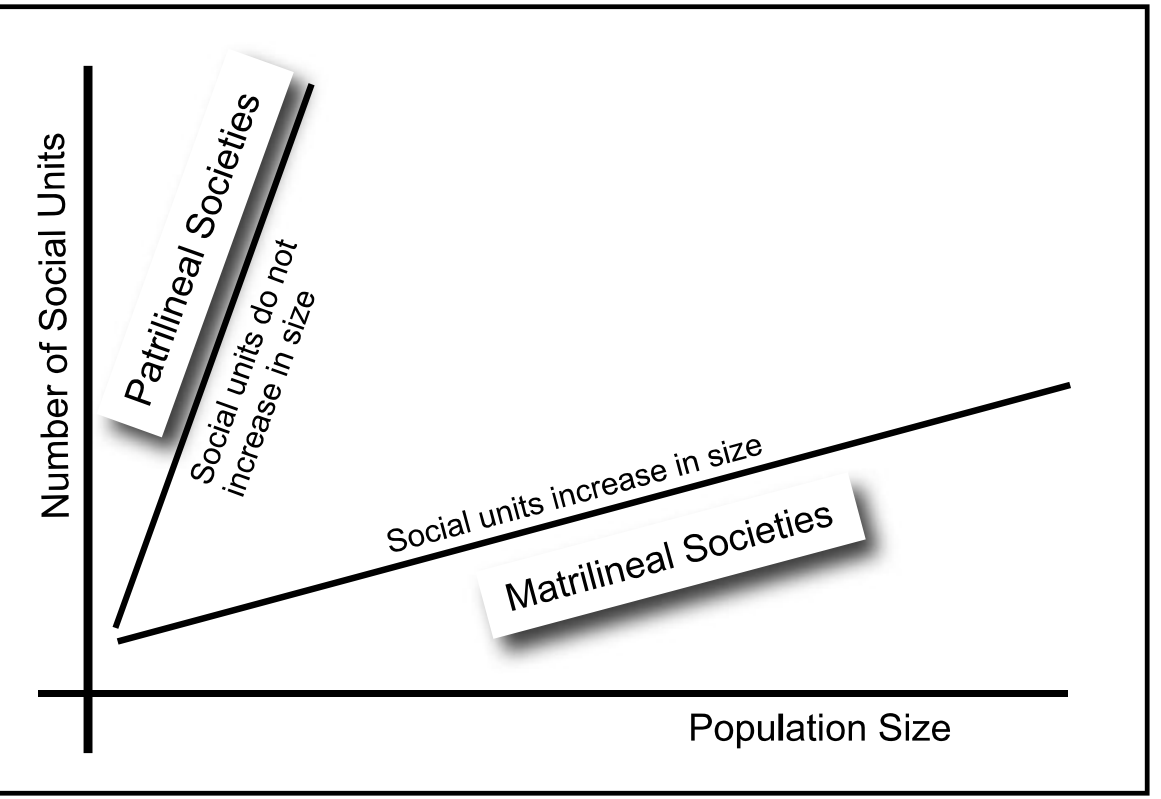

Fig. 5 Predicted relationship between number of social units, size of social units and population size for patrilineal and matrilineal societies

be relatively slow growth in the number of communities. Matrilineal societies are a canonical example of Spatial Pattern 2.

We make the following predictions.

Prediction 3 Societies will vary from small to large and community sizes will vary from small to large communities.

Prediction 4 Administrative hierarchical complexity will be deep since social units require an administrative hierarchy for maintaining internal cohesion of social units both within and between communities.

We also make the following predictions for the patrilineal, Spatial Pattern 2 and matrilineal, Spatial Pattern 3 societies (see Fig. 5).

Prediction 5 A graph of society population size against number of social units will have two components:

(a) a Spatial Pattern 2 component composed of patrilineal societies with a large, positive slope, relatively small social units independent of the population size, and no society with a large, total population size and

(b) a Spatial Pattern 3 component composed of matrilineal societies with a small, positive slope, size of social units increasing with the population size, and some societies with large, total population sizes 
Prediction 6 Any exceptions to Predictions 1-5 should have historical explanation for the exceptional status.

\subsection{Verification of the predictions}

The database for testing these predictions is a data set compiled by Henry Wright (2006) for paramount states and is shown in Table 1. The paramount states split bimodally into two groups based on Total Complexity. Patrilineal and matrilineal forms of social organization, with two exceptions, characterize these two groups. The two exceptions are Kom and Benin.

Kom is a patrilineal society that became matrilineal: "Chilver and Kaberry (1967, p. 31) also argued that the Kom were a patrilineal people ... who only adopted matrilineal institutions when they settled among peoples with matrilineal institutions. Nkwi (1973, p. 85) was very categorical in stating that the fact that 'rights and duties derived from the father preponderate over those derived from the mother, ... [this] leads me to assert that Kom was formerly a patrilineal society." "(Vubo 2005, p. 146). Thus its exceptional status has historical explanation.

Benin, though patrilineal, developed a standing army in the 1300's AD as a means for what was otherwise a relative weak central administration to gain control over trade and to reduce the autonomy of villages. Rather than an administrative structure developing from within, it was an administrative structure "imposed upon the social and economic basis of the society" (Sargent 1986, p. 402) and thereby transformed a patrilineal spatial structure of more-or-less autonomous local social units to a matrilineal-like system of administrative control over local social units. Its exceptional status also has historical explanation. This verifies Prediction 6.

With the historical explanation for Kom and Benin in place, the bimodal distribution for Total Complexity and patrilineal versus matrilineal (see right columns, Table 1) verifies Predictions 2 and 4. A graph of Number of Units versus Population size (see Fig. 6) verifies Prediction 5. The mean size of units for the patrilineal, Spatial 2 societies and the low standard error for the mean (see Fig. 6) verify Prediction 1. The mean size of units for the matrilineal, Spatial 3 societies and the large standard error for the mean (see Fig. 6) verify Prediction 3.

\section{Conclusions}

The simulation shows that even a simple, culturally framed agent model for interbirth spacing in small scale, hunter-gatherer societies leads to insights not previously recognized. Conventional models assume homogeneity across agents, leading to either assuming population growth is always a prime mover or, contradictorily, that equilibrium is the default condition for pre-state societies (see discussion in Read and LeBlanc 2003). The simulation model developed here shows both assumptions are inadequate, not for reasons relating to lack of empirical evidence, but due to failure to model behavior in accord with decision making that takes into account both cognitive/biological and cultural factors. 


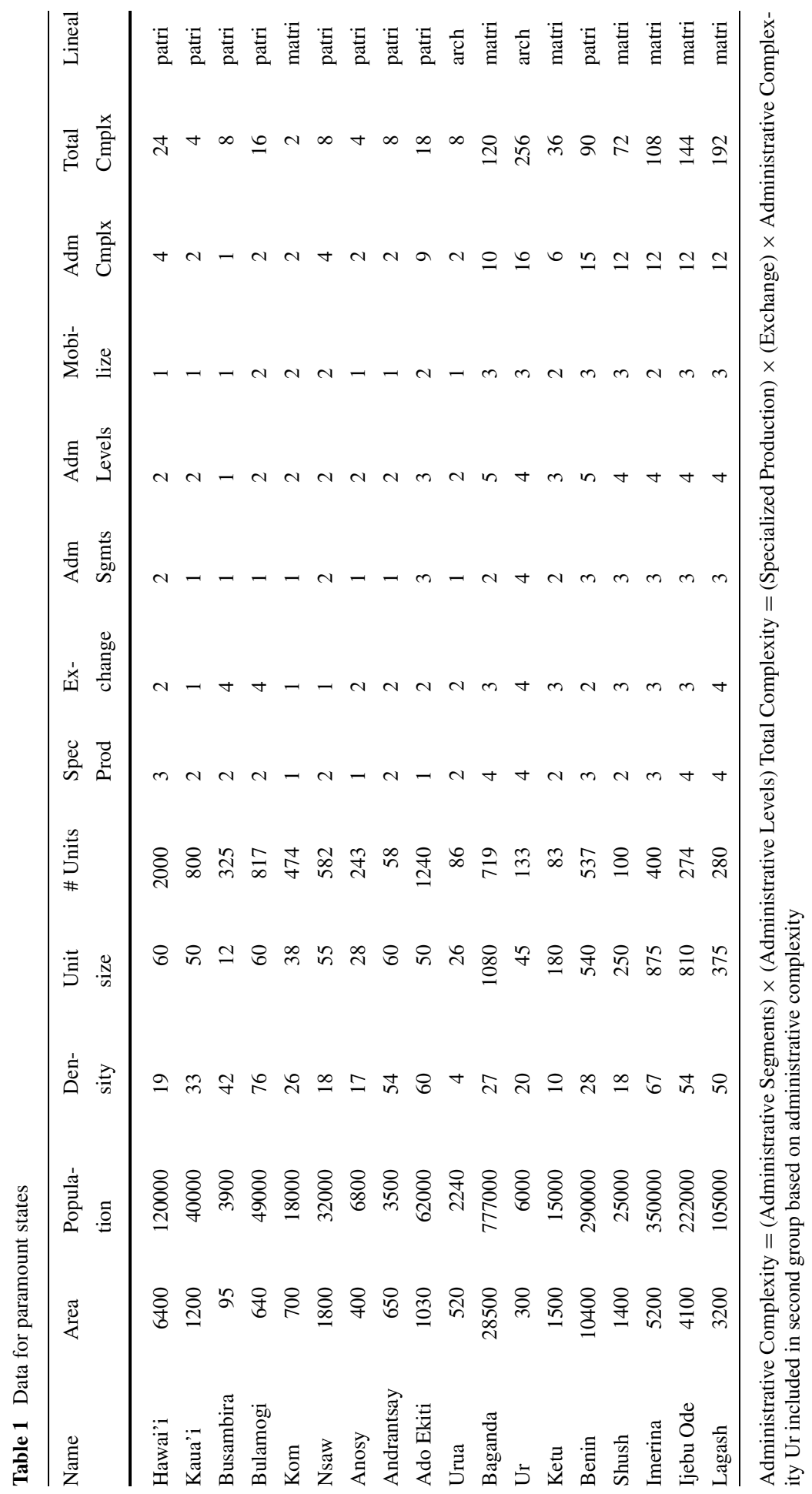




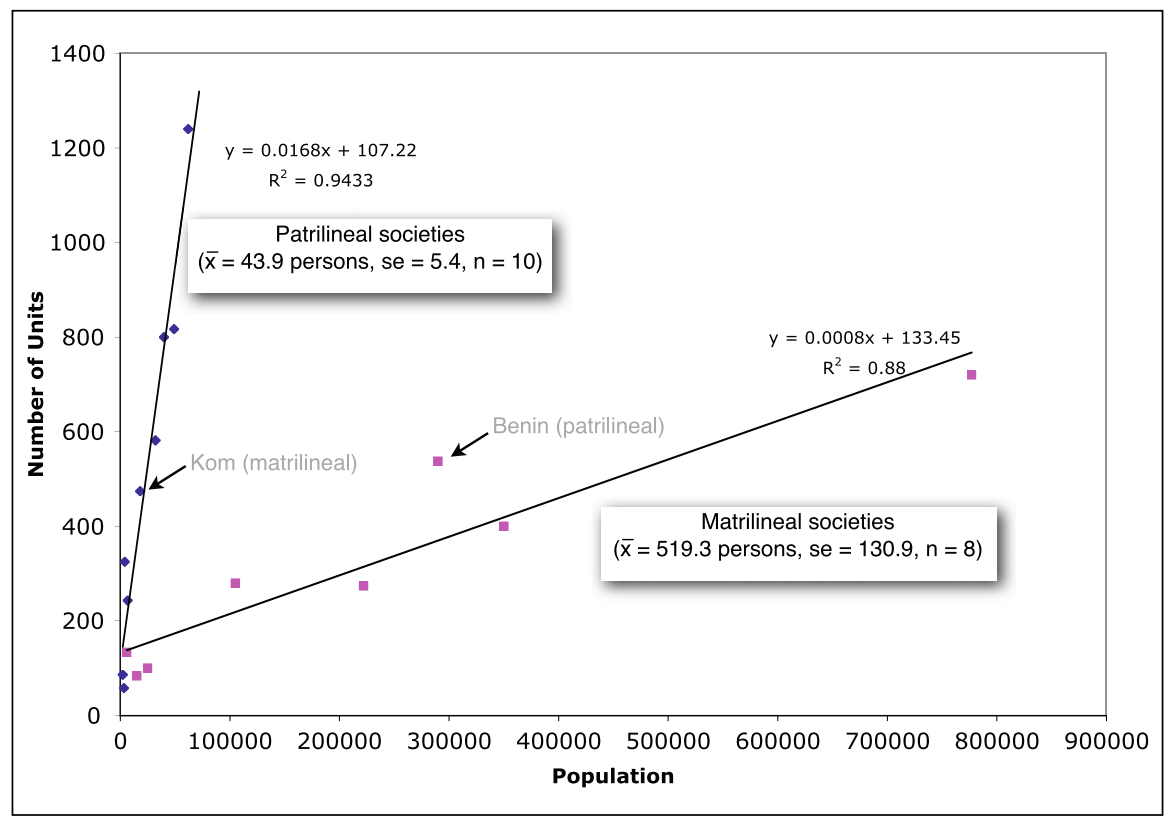

Fig. 6 Graph of number of social units for population size. Data divide into two groups: patrilineal and matrilineal societies. The groups, curves, and summary statistics match predictions. Data are from Wright (2006)

Though initiated on a small-scale society, the model has unexpected predictions for larger scale societies with different modes of social organization. It is applicable to societies at a different scale because decision-making by women that affects her reproductive potential is a constant feature across all societies and it is her realized fecundity that is the primary factor in demographic patterns. Universally, women are concerned with the health and well-being of their children and make decisions accordingly within a cultural framework of what is meant by health and well-being. But such decisions are at the level of individual agents who need not be in synchrony with regard to decisions made individually. One !Kung san woman may decide to become pregnant while at the same time another woman may continue to nurse a child and thereby defer a pregnancy, both acting according to the same decision model because, in this case, the environment in which decision making is made has been modified both internally by a woman's past decisions and externally by decisions made by other women. One woman may no longer have an infant and so has the time/energy for a new child because food resources are still plentiful, given the current population density, whereas the other woman is still nursing an infant.

In order to apply the same birth spacing model to other societies with other forms of social organization we only need to take into account differences that might arise over the feedback, if any, a woman receives as a result of changes in population size (or density) in the society in which she is member. Unexpected, though, was the unambiguous support for the model predictions. The pattern shown in Fig. 6 needs no statistical analysis and underscores the fact that phenomena, seemingly complex 
on the surface, may have relative simple, underlying structuring processes. It is here where the power of multi-agent simulations lies. Multi-agent simulations have the ability to make sense of complex phenomena through realistic simulations formulated in accord with the processes, both biological and cultural, that underlie the decisions we make, while taking into account the consequences of agent interactions have on the conditions under which those decisions are made. Multi-agent simulations developed in this manner will be ones in which the human aspect of human societies takes center stage.

Open Access This article is distributed under the terms of the Creative Commons Attribution Noncommercial License which permits any noncommercial use, distribution, and reproduction in any medium, provided the original author(s) and source are credited.

\section{References}

Axelrod R, Hammond RA (2003) The evolution of ethnocentric behavior. Paper presented at the Midwest Political Science Convention, April 3-6, 2003, Chicago, IL. Available via http://www.personal.umich.edu/ axe/research/AxHamm_Ethno.pdf

Binford L (2001) Constructing frames of reference: an analytical method for archaeological theory building using ethnographic and environmental data sets. University of California Press, Berkeley

Caminada M (2009) Truth, lies and bullshit: distinguishing classes of dishonesty. Paper given at the social simulation workshop at the international joint conference on artificial intelligence 2009, http://ss-ijcai2009.di.fc.ul.pt/programme.html, pp 1-12

Chilver E, Kaberry PM (1967) Traditional Bamenda. The precolonial history and ethnography of the Bamenda grassfields, vol I. Ministry of primary education and social welfare and West Cameroon antiquities commission, Buea

Clark AV, Fossett M (2008) Understanding the social context of the Schelling segregation model. Proc Natl Acad Sci, USA 105:4109-4114

Fossett M (2006) Ethnic preferences, social distance dynamics, and residential segregation: theoretical explorations using simulation analysis. J Math Sociol 30:185-273

Fridman N, Kaminka GA, Traub M (2009) First steps towards a social comparison model of crowds. Paper given at the social simulation workshop at the international joint conference on artificial intelligence 2009, pp 1-12. http://ss-ijcai2009.di.fc.ul.pt/programme.html

Garcia-Valverde T, Serrano E, Botia JA, Gomez-Skarmeta A, Cadenas JM (2009) Social simulation to simulate societies of users immersed in an Ambient Intelligence Environment. Paper given at the social simulation workshop at the international joint conference on artificial intelligence 2009, pp 1-12. http://ss-ijcai2009.di.fc.ul.pt/programme.html

Gilbert N (2007) Computational social science: agent-based simulation. In: Phan D, Amblard F (eds) Agent-based modeling and simulation in the social and human sciences. The Bardwell Press, Oxford, pp 115-134

Knauft BM (1996) The human evolution of cooperative interest. In: Gregor T (ed) A natural history of peace. Vanderbilt University Press, Nashville, pp 71-94

Lakkaraju K, Swarup S, Gasser L (2009) Consensus under constraints: modeling the Great English Vowel Shift. Paper given at the social simulation workshop at the international joint conference on artificial intelligence 2009, pp 1-12. http://ss-ijcai2009.di.fc.ul.pt/programme.html

Marshall L (1976) The !Kung of Nyae Nyae. Harvard University Press, Cambridge

Martin N (2008) Homo socionicus: a case study of simulation models of norms. J Artif Soc Soc Simul 11. http://jasss.soc.surrey.ac.uk/11/4/6.html

Németh A, Takács K (2007) The evolution of altruism in spatially structured populations. J Artif Soc Soc Simul 10. http://jasss.soc.surrey.ac.uk/10/3/4.html

Nkwi PN (1973) A clanship study: a systematic study of clans in Kom. Unpublished mimeograph, Faculté des Lettres, Fribourg

O’Sullivan D (2008) Geographical information science: agent-based models. Prog Hum Geogr 32:541550 
Page S (2008) Review of "Social simulation: technologies, advances and new discoveries" by Bruce Edmonds, Cesareo Hernandez and Klaus G Troitzsch. J Artif Soc Soc Simul 11. http://jasss. soc.surrey.ac.uk/11/4/6.html

Read D (1998) Kinship based demographic simulation of societal processes. J Artif Soc Soc Simul 1. http://www.soc.surrey.ac.uk/JASSS/1/1/1.html

Read D (2003) Emergent properties in small-scale societies. Artif Life 9:419-434

Read D, LeBlanc S (2003) Population growth, carrying capacity, and conflict. Curr Anthropol 44:59-85

Sargent RA (1986) From a redistribution to an imperial social formation: Benin c1293-1536. Can J Afr Stud 20:402-427

Taçon P, Chippindale C (1994) Australia's ancient warriors: changing depictions of fighting in the rock art of Arnhem Land, N.T. Camb Archaeol J 4:211-248

Vubo EY (2005) Matriliny and patriliny between cohabitation-equilibrium and modernity in Cameroon grassfields. Afr Study Monogr 26:145-182

Wilensky U (1999) NetLogo. Center for connected learning and computer-based modeling, Northwestern University, Evanston

Wilensky U (2006) NetLogo ethnocentrism model. Center for connected learning and computer-based modeling, Northwestern University, Evanston

Wright HT (2006) Atlas of chiefdoms and early states. Struct Dyn eJ Anthropol Relat Sci 1

Zappala J, Logan B (2009) Effects of resource availability on consensus decision making in primates. Paper given at the social simulation workshop at the international joint conference on artificial intelligence 2009, pp 1-12. http://ss-ijcai2009.di.fc.ul.pt/programme.html

Zhang Y, Leezer J (2009) A reinforcement and memory-based model of human-like behavior. Paper given at the social simulation workshop at the international joint conference on artificial intelligence 2009, pp 1-12. http://ss-ijcai2009.di.fc.ul.pt/programme.html

Zubrinsky C, Bobo L (1996) Prismatic metropolis: race and residential segregation in the City of Angels. Soc Sci Res 25:335-374

Dwight Read received his Ph.D at UCLA in mathematics, doing research on properties of abstract algebras. He is a Professor of Anthropology and of Statistics and publishes in all the subdisciplines of anthropology (transition from biological to cultural evolution, theory and method of artifact classification, mathematical representation of cultural constructs, especially kinship terminologies). His current research focuses on the interrelationship between the material and the ideational domains in human societies. He had a Visiting Scientist affiliation with the IBM Los Angeles Research Center from 1986-1989. He has edited two Special Issues of the Journal of Quantitative Anthropology and a Special Issue of the Journal of Artificial Societies and Social. He has developed a major computer program (Kinship Algebraic Expert System, or $K A E S$ ) that constructs a formal (algebraic) model for the logic underlying the structure of a kinship terminology. 